\title{
Monitoring and Surveillance of Hemodialysis Access
}

\author{
Nischal Koirala, MS ${ }^{1,2}$ Evamaria Anvari, MD ${ }^{3}$ Gordon McLennan, MD, FSIR² \\ ${ }^{1}$ Department of Chemical and Biomedical Engineering, Cleveland \\ State University, Cleveland, Ohio \\ 2 Department of Biomedical Engineering, Cleveland Clinic, Cleveland, \\ Ohio \\ ${ }^{3}$ Department of Nephrology and Hypertension, Cleveland Clinic, \\ Cleveland, Ohio \\ Address for correspondence Gordon McLennan, MD, FSIR,
Department of Biomedical Engineering, 9500 Euclid Avenue, ND3,
Cleveland Clinic, Cleveland, OH 44195 (e-mail: mclenng@ccf.org).
}

Semin Intervent Radiol 2016;33:25-30

\begin{abstract}
Keywords

- vascular access

- monitoring

- surveillance

- arteriovenous fistula

- arteriovenous graft

- interventional radiology

Access surveillance using invasive or noninvasive methods with an objective to improve access patency and decrease hospital admissions for access dysfunction in dialysis population has been promoted, but its success to predict incipient thrombosis and subsequent access failure is a controversial topic. Some studies have shown improvement in access outcomes, while others have failed to demonstrate an ideal method to diagnose access problems. Furthermore, the use of endovascular interventions such as percutaneous transluminal angioplasty to timely correct access problem might itself be a promoter of neointimal hyperplasia and restenosis during balloon angioplasty. There are significant costs and efforts associated with routine dialysis surveillance; therefore, it is necessary to understand whether such programs will help improve access-related problems and guarantee adequate dialysis care. It is generally agreed upon that despite the lack of guaranteed success of surveillance, such strategies have helped improve dialysis management, resulted in decreased costs and hospitalizations, and represented clinically relevant indications of failure prior to planning any radiological or surgical intervention. In this study, the authors review monitoring and surveillance measures in place, and their associated merits and limitations to detect stenosis and prevent incidences of vascular access thrombosis.
\end{abstract}

Objectives: Upon completion of this article, the reader will be able to discuss the current monitoring and surveillance methods, and outcomes of such methods, for hemodialysis AV accesses.

Accreditation: This activity has been planned and implemented in accordance with the Essential Areas and Policies of the Accreditation Council for Continuing Medical Education (ACCME) through the joint providership of Tufts University School of Medicine (TUSM) and Thieme Medical Publishers, New York. TUSM is accredited by the ACCME to provide continuing medical education for physicians.

Credit: Tufts University School of Medicine designates this journal-based CME activity for a maximum of 1 AMA PRA Category 1 Credit $^{\mathrm{TM}}$. Physicians should claim only the credit commensurate with the extent of their participation in the activity.

The prevalence of end-stage renal disease (ESRD) has increased in the United States leading to a decrease in the quality of life for patients and an overburdened budget associated with medical care. ${ }^{1}$ Incidence of ESRD rose steadily from 1980 to 2010, and has plateaued or declined since then. However, at the end of 2012, more than 636,905 people received treatment for ESRD which represents a $1.3 \%$ increase from 2011. ESRD has incurred more than $\$ 28.6$ billion in health care expenditure. ${ }^{1}$ Additionally, the prevalent dialysis population increased by $3.8 \%$ in 2012.
Issue Theme Dialysis Interventions; Guest Editor, Gordon McLennan, MD, FSIR
Copyright (c) 2016 by Thieme Medical Publishers, Inc., 333 Seventh Avenue, New York, NY 10001, USA. Tel: +1(212) 584-4662.
DOI http://dx.doi.org/ 10.1055/s-0036-1572548. ISSN 0739-9529. 
For hemodialysis, the vascular system needs to be accessed. The methods currently available for access include using a central venous catheter (CVC); insertion of a graft to connect an artery and a vein (arteriovenous graft [AVG]), preferably at the arm, or surgically creating a connection between an artery and vein (arteriovenous fistula [AVF]). Fistula is the preferred method or "gold standard" for dialysis access due to longer patency, fewer infections, and no immune challenge because of the lack of nonnative tissue. However, full maturation of fistulae takes 4 to 8 weeks, and not every patient has the luxury to wait that long to receive treatment. The National Kidney Foundation's Kidney Disease Outcomes Quality Initiative (KDOQI) ${ }^{2}$ and Fistula First Catheter Last Initiative suggests the prevalent use of fistula and the minimal use of CVC for access to improve hemodialysis outcomes.

An AV graft uses a synthetic biocompatible material such as polytetrafluoroethylene or even cadaveric tissue to make a conduit for connecting the vessels; the use of the former is far more common. Grafts can be used in patients who are not eligible to receive a fistula due to smaller or inaccessible veins, and are relatively permanent and therefore are the second preferred method of access. AV grafts are marked by immunological challenges, clots, and infections. The CVC method uses dual lumen catheters placed vie the internal jugular, chest, or groin veins that draw impure blood from the systemic circulation and returns filtered blood from the dialyzer back into the venous circulation. Placement of CVC is generally quick and easy, and can be used in all patients in emergency situations. However, CVCs are prone to clot formation, lower access duration, and infections.

The choice of using an AV fistula, AV graft, or CVC is dependent on the urgency of dialysis, and the patient's condition. The presence of stenosis in the access circuit reduces the intra-access flow or elevated pressure in the venous outflow, which can be interpreted as a sign of imminent access failure. The cause of a stenosis due to neointimal hyperplasia at the venous anastomosis may be due to hemodynamic factors such as wall shear stress. Additionally, stenosis in the access leading to thrombosis is the major cause for access dysfunction. The purpose of surveillance is to detect access dysfunction and perform interventions prior to access thrombosis in the hopes of achieving better clinical outcomes. The following sections will discuss the current methods available for monitoring and surveillance of dialysis access and limitations associated with the various access circuits.

\section{Management Techniques for Hemodynamic Access}

Two mechanisms have been broadly identified for the management of hemodynamic access: monitoring and surveillance. Monitoring includes physical examination at dialysis care centers to initially evaluate the patient's access status. Surveillance includes all the clinical procedures performed to evaluate the intra-access flow, directly or indirectly, for detailed symptoms of dysfunction. The direct method of examination includes duplex ultrasound imaging and magnetic resonance angiography (MRA) $)^{3,4}$; the indirect method includes the use of ultrasound dilution, ${ }^{5,6}$ conductivity, ${ }^{7}$ temperature, ${ }^{8}$ or optical methods. ${ }^{9,10}$

\section{Physical Examination}

Physical examination must be carefully performed, and is always the first step used to diagnose and treat a malfunctioning access. A combined approach of physical exam and clinical evaluation must be performed instead of solely relying on technological results. Studies ${ }^{11,12}$ comparing physical exam to angiography have demonstrated that physical examination can accurately detect and localize a stenosis in AVF and AVG. KDOQI guidelines recommend physical examination (by a qualified professional) of the fistula at least once every month to detect any signs of abnormality or malfunction. ${ }^{2}$ Physical examination include inspection, palpation, and auscultation. Inspection includes examining the access sites for the presence of any edema, swelling, or bruises. Visualization of multiple collaterals on the arm or chest region indicates the presence of a central stenosis. Palpation includes assessing for a thrill at the access site. Auscultation is listening for a bruit, which is normal in a functioning access. Other symptoms to consider include pain in the hand and prolonged bleeding during or after needle insertion. - Table 1 lists the components of physical exam, ${ }^{13,14}$ while - Table 2 demonstrates the signs of possible stenosis during physical exam. ${ }^{13,14}$

\section{Patient Education}

It has also been suggested that patient education is vital to graft survival. Care providers should educate the patient on how to assess their AVG or AVF by teaching them the signs and symptoms that indicate access dysfunction. Such educations

Table 1 Physical examination (Look, Listen, and Touch)

\begin{tabular}{|l|l|}
\hline Exam type & Exam details \\
\hline Inspection (“Look”) & $\begin{array}{l}\text { Swelling, pain, aneurysm, edema, presence of multiple collateral vessels, fluid leakage, bleeding, } \\
\text { color (redness), steal syndrome symptoms } \\
\text { When arm is elevated, the fistula collapses completely; when there is a stenosis, } \\
\text { the fistula remains distended }\end{array}$ \\
\hline Auscultation (“Listen') & Sound of access (bruit) \\
\hline Palpation (“Touch”) & Presence and intensity of pulse/thrill \\
\hline
\end{tabular}


Table 2 Physical exam suggesting venous stenosis

\begin{tabular}{|l|l|l|}
\hline Feature & Normal & Stenosis \\
\hline Thrill & Only at arterial anastomosis & At site of stenotic lesion \\
\hline Pulse & Not pulsatile, soft and easily compressible & $\begin{array}{l}\text { Becomes pulsatile } \\
\text { Difficult to compress }\end{array}$ \\
\hline Bruit & Low pitch, continuous in systole and diastole & High pitch, discontinuous, and noted in systole only \\
\hline
\end{tabular}

may improve the patency of hemodynamic access. Education includes teaching patients to look for signs of infections, arm swelling, maintaining personal hygiene, feeling pulse/thrills or listen for bruit, and to take care of the vascular access arm area by refraining from lifting heavy loads, not allowing intravenous (IV) access or blood pressure check on that arm, and to avoid sleeping on or otherwise putting direct pressure over the access.

\section{Surveillance}

Surveillance methods include measurements that use clinical tools such as imaging (Doppler ultrasound ${ }^{15-17}$ or MRA), ultrasound dilution methods, ${ }^{5,6}$ recirculation, ${ }^{18}$ or pressure measurements. ${ }^{19,20}$ Other methods based on blood properties such as electrical conductivity, ${ }^{7}$ optical properties, ${ }^{9,10}$ or temperature $^{8}$ (thermodilution) are also available for use. These methods provide anatomical imaging, pressure information, flow rate, or dialysis adequacy that help evaluate the access status.

\section{Imaging}

Imaging techniques include evaluation of the access by duplex ultrasound imaging or from MRA. Compared with duplex ultrasound, MRA provides high-quality imaging that is important for stenosis diagnosis. However, these methods are time consuming, costly, and cannot be used during dialysis. They provide intra-access blood flow information and access status through anatomical imaging. A decrease in intra-access flow or obstruction in access from anatomical images is typically an indication of stenosis.

The Duplex ultrasound method requires knowledge of the accurate cross-sectional area of the access, which is dependent on the operator and subject to error due to the chosen angle of insonation. It also provides an anatomical assessment of the access, which is crucial for detecting an underlying stenosis.

\section{Pressure Measurement}

Pressure measurement is another technique for AVG or AVF surveillance to assess impending access failure. The relationship between access flow and pressure can be expressed as follows:

$$
\text { Flow }\left(\mathrm{Q}_{\mathrm{A}}\right)=\text { Pressure gradient }(\Delta \mathrm{P}) / \text { Resistance }(\mathrm{R})
$$

Where $\Delta \mathrm{P}$ is the gradient between the fistula artery and central vein, and $R$ is the resistance in the access.
The presence of a stenosis causes a reduction in flow in the venous outflow, while a concurrent increase in pressure in the arterial inflow is noted. Observing a change in pressure can predict a change in flow; hence, a stenosis can be predicted. Direct venous pressure (DVP) measurement and static pressure measurement are the two pressure parameters used in the calculation of flow. Dynamic venous pressure is measured in the presence of extracorporeal blood. DVP can be measured at the beginning of dialysis because it requires low blood flow rates $(50-225 \mathrm{~mL} / \mathrm{min}$ ) and uses 15 -gauge needles to measure blood flow. The baseline pressure reading should be close to 124 to $150 \mathrm{~mm} \mathrm{Hg}$, depending on different machines and facility-specific baselines. Three consecutive readings greater than $150 \mathrm{~mm} \mathrm{Hg}$ should be considered abnormal and the patient referred to further access evaluation. However, this method does not produce consistent results owing to changes in needle size, machine type, and blood flow. At higher blood flow rates, resistance is from needles and not from the access, making the measurement less reliable for these reasons. The KDOQI guidelines do not recommend using the unstandardized DVP as an indicator for access stenosis. ${ }^{2}$

Static pressure measurement is the KDOQI-recommended technique for access surveillance. It is performed prior to dialysis (in the absence of extracorporeal blood flow), and provides flow information based on pressure change due to flow resistance. It eliminates variables of DVP such as needle size, machine type, and blood flow, and is therefore more reliable. It is corrected for differences in the height between the venous needle in the access and venous drip chamber, and normalized for mean arterial pressure (MAP). The normalized ratio of intra-access pressure (IAP) at the arterial and venous end to the MAP is calculated as follows:

$$
\begin{aligned}
& \text { Arterial ratio }=(\text { arterial IAP }+ \text { arterial height correction }) / \\
& \text { MAP } \\
& \text { Venous ratio }=(\text { venous IAP }+ \text { venous height correction }) / \\
& \text { MAP }
\end{aligned}
$$

An arterial segment static pressure ratio $>0.75$ in grafts or a venous segment static pressure ratio $>0.5$ in grafts or fistulae should be referred for further examination.

\section{Intra-Access Flow Measurements}

Intra-access flow measurements include ultrasound dilution 5,6 (Transonic $^{\circledR}$, Ithaca, NY) glucose pump infusion, ${ }^{21}$ transcutaneous flow monitor, ${ }^{22}$ conductivity dialysance, ${ }^{23,24}$ and 
variable flow Doppler ultrasound (VFDU). ${ }^{25,26}$ The intraaccess flow measurements based on indicator dilution theory that require exchange of needles for flow measurement include ultrasound dilution, thermodilution, or conductivity dialysance. Measurement systems include software that calculates area under the curve as a measure of recirculation to calculate flow. Newly developed methods such as the glucose pump test (GPT) (based on indicator dilution theory), transcutaneous flow monitors, or variable pump Doppler flow do not require reversal of dialysis lines.

\section{Ultrasound Dilution (Transonic ${ }^{\circledR}$ )}

The ultrasound dilution method, also known as the Krivitski method, ${ }^{5,6}$ is the most commonly used technique to measure intra-access flow rates during dialysis. It is commercially available; patients with AVG are more commonly assessed using this method; however, studies ${ }^{27,28}$ have reported that it may also be used for AV fistulae. It involves detection of ultrasound wave velocity through the blood as a result of dilution; this is performed by injecting a saline bolus when the dialysis lines are reversed. This method requires special monitors, flow/dilution sensors, and a software package to calculate the flow rate. A temporary recirculation is created by reversing the dialysis flow lines (the arterial needle connects to the venous line and vice versa), with sensor clips attached at each line in the extracorporeal circuit. A calibration sensor is placed at the venous line and a flow/dilution sensor is clipped to the arterial line. Saline is injected into the venous line, and dilution information as a result of mixing of blood with the saline is collected by the sensor at the arterial line. From these data, the vascular access flow $\left(Q_{a}\right)$ is calculated by the software from the area under the curve as a measure of recirculation based on the following equation:

$$
\mathrm{Q}_{\mathrm{a}}=\mathrm{Qb}_{\mathrm{b}}\left(\frac{1}{\mathrm{R}}-1\right)(1)
$$

Where $\mathrm{Q}_{\mathrm{b}}$ is blood pump flow and $\mathrm{R}$ is the access recirculation caused by the reversal of dialyzer bloodlines and calculated from the ratio of areas under dilution curves recorded by the venous and arterial sensors. This method requires thorough mixing of the saline, and does not consider error introduced by a second pass of the saline indicator due to cardiopulmonary recirculation. Error arising from incorporating cardiopulmonary recirculation into the primary signal is estimated as $Q_{a}$ /cardiac output, which increases as $Q_{a}$ increases, and will underestimate real access flow. ${ }^{29}$

The flow procedure should be repeated at least three times for a reliable measurement, and the information needs to be recorded routinely over a period of time. Any fluctuation in the flow rate is an indicator of a potential access problem. KDOQI suggests that the low flow rate threshold in AVG is a rate $<600 \mathrm{~mL} / \mathrm{min}$, while in AVF $<400$ to $500 \mathrm{~mL} / \mathrm{min}$ is considered abnormal. Also, if a baseline access flow of $1,000 \mathrm{~mL} / \mathrm{min}$ has decreased by $25 \%$ over a period of $\leq 4$ months, a fistulogram should be performed. ${ }^{2}$ When available at the dialysis units, Transonics are used on a monthly basis.

\section{Online Dialysance Conductivity}

Online (real-time) measurement of dialysate sodium conductivity provides an alternative measure of effective urea clearance during hemodialysis. Gotch et $\mathrm{al}^{23}$ and Mercadal et $\mathrm{al}^{24}$ proposed a technique used to measure access blood flow based on conductivity.

Owing to similar molecular weights and diffusion of sodium chloride and urea, dialysance of ionized sodium is nearly the same as urea, thereby obsoleting the need to remove samples or requiring additional equipment for measuring urea clearance. Change in dialysate electrical conductivity is a measure of urea diffusion across the dialyzer membrane. Dialysate conductivity is measured by placing the conductivity probes on the dialyzer inlet and outlet lines from the access, and provides a measure of ion flux during dialysis. Ionic dialysance is calculated by assuming that the patient's plasma conductivity at the inlet port remains constant during the short time needed to make measurements. By changing the dialysate/water proportion, a significant electrolyte gradient is established between the blood and the dialysate, which results in a conductivity change from the inlet to outlet port. This is a function of sodium dialysance and reflects movement of electrolytes into the patient.

Access blood flow $\left(Q_{A}\right)$ from dialysance can be calculated with blood lines in either their normal position (D) or reverse position $\left(D_{\text {rev }}\right)$. This method assumes no access recirculation

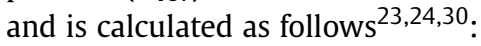

$$
\mathrm{Q}_{\mathrm{A}}=\frac{\left(\mathrm{D}-\mathrm{Q}_{\mathrm{F}}\right) \mathrm{D}_{\mathrm{rer}}}{\mathrm{D}-\mathrm{D}_{\mathrm{TeV}}}(2)
$$

For $\mathrm{Q}_{\mathrm{F}}=0$ (no ultrafiltration), Eq. (2) results into

$$
\mathrm{Q}_{\mathrm{A}}=\frac{\mathrm{D} \cdot \mathrm{D}_{\mathrm{rev}}}{\mathrm{D}-\mathrm{D}_{\mathrm{req}}}(3)
$$

where $Q_{F}$ is the blood flow in dialysis circuit.

\section{Variable Flow Doppler Ultrasound}

VFDU $^{25}$ has been developed as a newer technique to eliminate the errors introduced by cross-sectional area measurements or knowledge of the beam angle used for Duplex ultrasound. It also does not require reversal in dialysis flow lines as with UD methods (which are time consuming). The Doppler signal is proportional to volume flow and adjusts itself for beam angle variance or changes in pump speed, making this method more accurate than many other methods. The flow rate is calculated from the change in Doppler signal in the access using variable pump rates. This method uses two needles introduced into the access for collecting the blood from arterial side (QA), to feed into the dialysis pump circuit (QB), and back to the venous stream from the other needle. The dialysis blood pump circuits form a parallel circuit with the access. The Doppler signal is measured in the access between the two needles $(\mathrm{QR})$ as a function of the pump rate, which is calculated as $Q R=Q A-Q B$. Using various values of pump speed, a function $S=F(Q B)$ is modeled and the intercept at which ${ }_{Q} R=0$ is calculated. This gives the Eq. $0=\mathrm{QA}-\mathrm{QB}$, which means that $\mathrm{QB}=\mathrm{QA}$; this value is the access flow rate. The transducers and software for modeling are produced by Specs USA (Houston, TX). The method of intraaccess flow determined by this method is not popular, and the 
accuracy of $\mathrm{QB}$ as a function of variable pump speed is also an issue.

\section{Glucose Pump Test}

The GPT ${ }^{21}$ is a method of access blood flow determination that uses a constant glucose bolus into the arterial line, and withdrawal of blood from the venous line before and after the infusion. By knowing the concentration of the infused glucose concentration $\left(C_{i}\right)$, and measurement of glucose levels before $\left(\mathrm{C}_{\mathrm{a} 1}\right)$ and during infusion $\left(\mathrm{C}_{\mathrm{a} 2}\right)$ by using bedside glucometer, the access blood flow $\left(Q_{a}\right)$ can be calculated as follows:

$$
Q_{a}=Q_{i} \times \frac{\left(C_{i}-C_{a 2}\right)}{\left(C_{a 2}-C_{a 1}\right)}(4)
$$

Where $\mathrm{Q}_{\mathrm{i}}$ is the pump infusion rate. This method is quick, economical, and easy to perform. It does not require reversal of blood lines as with other methods such as ultrasound dilution. It is based on changes in the glucose dilution as it is infused into the access compared with the baseline glucose level in the access (before glucose bolus was infused).

\section{Transcutaneous Access Flow Monitor}

Transcutaneous access flow monitor uses an optical sensor that is placed on the skin immediately above the access. This method measures the hematocrit concentration in the blood by the properties of reflection of light by red cells. This method also does not require reversal of dialysis lines. An indicator fluid is injected into the venous line that mixes with blood, and effectively reduces changing the hematocrit concentration; this is recorded over time by the hematocrit sensor. The difference in hematocrit concentration is used to calculate the intra-access blood flow. ${ }^{22}$

\section{Thermodilution}

The thermodilution technique ${ }^{8}$ uses the change in dialysate temperature when mixed with blood to calculate the intraaccess blood flow rate. Temperature-sensitive thermistors are placed in the arterial and venous lines, which detect changes in temperature downstream over a period of time when cold injectate (indicator) of known volume and temperature is introduced in the access. Blood temperature monitor measures the change in temperatures, and the flow rate is calculated from the mean blood temperature and the duration of transit of the injectate (i.e., area under the curve).

\section{Access Recirculation}

Access recirculation refers to return of the dialyzed blood immediately back to the dialysis machine instead of entering back into the patient's vasculature. Turbulent nonlinear flow and recirculation usually signifies the presence of a stenosis upstream of access. This recirculation attributes to dialysis inefficiency and is usually a sign of an access abnormality. Recirculation may also result due to incorrect placement of bloodline needles or inadequate spacing of needles, so false positives can occur. On the other hand, the presence of a significant mid-access stenosis is usually not detected by conventional methods, as no recirculation is observed: there is sufficient blood flows through the extracorporeal circuit and back into the venous limb to bypass the intra-access resistance. Venous stenosis is one of the most common causes of recirculation, as the blood that reenters the venous limb cannot exit the access, resulting in the same blood being redialyzed. Access recirculation is measured by urea-based methods, blood temperature monitoring, or indicator dilution methods.

\section{Urea Dilution}

Measuring the reversal in urea concentration after reversing the lines is another method that does not require injection of saline for measuring the access flow. The dialysis arterial line has more urea concentration compared with the venous line; a reversal of these lines causes dilution of urea in the arterial line that is dependent on the degree of recirculation and inversely proportional to Qa. The access flow is calculated from the access blood water flow rate (Qaw) adjusted for hematocrit and protein. Qaw in relation to the effective urea clearance by the dialyzer $(\mathrm{K})$ is found from urea concentrations in the dialysate with needles in the normal $(\mathrm{Cn})$ and reverse $(\mathrm{Cr})$ positions. ${ }^{7,31,32}$ The following formula applies:

$$
\frac{\mathrm{F}}{\mathrm{Q}: 1 w}=\frac{(\mathrm{Cr}-\mathrm{Cr})}{\mathrm{Cr}}(5)
$$

The percentage recirculation ( $R$ ) based on the two-needle urea method is calculated from the concentration of blood urea nitrogen (BUN) sample from the peripheral (systemic) blood $(\mathrm{S})$, arterial $(\mathrm{A})$, and venous lines $(\mathrm{V})$.

$$
\mathrm{R}=\frac{S-\Lambda}{S-V} \times 100(6)
$$

The magnitude of access recirculation lies between 0 and 1 . If there is no recirculation, then the BUN from the peripheral and arterial line is the same $(S=A)$, and $R$ then equals 0 (no recirculation). However, in the extreme (worst) case of recirculation, $A=V$, and $R=1$ (complete recirculation), inferring that all the postdialyzed venous blood reenters the arterial line instead of the systemic circulation. From Eq. (4), it can be inferred that a lower concentration of BUN from the arterial line compared with systemic circulation indicates the presence of recirculation of dialyzed blood into the arterial line. The urea-based method to test recirculation is relatively insensitive at high flow, and works only for systems with relatively low blood flow. Any recirculation in excess of $10 \%$ by the ureabased method, or greater than $5 \%$ in non-urea-based method, is abnormal and requires further investigation. ${ }^{30,33}$

\section{Differential Conductivity}

Differential conductivity ${ }^{7}$ detects the access recirculation by measuring the changes in conductivity between the arterial and venous lines. A hemodynamic meter is an electromagnetic device that measures changes in conductivity in the access when a high conductivity indicator fluid is injected in the arterial line and withdrawn from the venous line. After the injection of the indicator fluid (hypertonic saline), it mixes with the blood, thereby altering the conductivity of the blood in the access. If recirculation is present, the altered conductivity is proportional to the degree of recirculation. 
The differences in the conductivities are used to quantitatively determine the recirculation in the access.

\section{Conclusion}

Though surveillance is proposed as a method to improve access patency and help intervene quickly to reduce mortality and morbidity associated with access failure, the benefits of access surveillance has not been universally demonstrated. Several other factors such as patient demographics may influence the risk of access thrombosis, making it difficult to achieve desirable sensitivity and specificity of surveillance methods. In fact, frequent catheter interventions for access status might lead to inflammatory reactions and restenosis at the insertion site, and reduce patency of the access. Vascular access is the "life line" in dialysis patients, and having a proper surveillance method to detect stenosis would be extremely helpful to reduce incidence of thrombosis and treat it promptly in an outpatient setting. Thorough physical exam combined with a clinical surveillance program by experienced health professionals should be the recommended procedure for evaluating dialysis patients for signs of access abnormalities. Some data suggest that the intra-access flow method is not sufficient to diagnose imminent thrombosis, and other methods such as duplex ultrasound are required to select patients who need to undergo interventional procedures. However, when implemented in a systematic way, monitoring and surveillance procedures may provide clinical indications for access failure that can lead to appropriate interventions to maintain access patency. Further studies are required based on the type of access, patient-specific factors, hospitalizations, and cost to determine the optimum method and frequency of access monitoring and surveillance.

\section{References}

1 Saran R, Li Y, Robinson B, et al. US renal data system 2014 annual data report: Epidemiology of kidney disease in the united states. Am J Kidney Dis 2015;66(1, Suppl 1):S1-S305

2 National Kidney Foundation (NKF). KDOQI clinical practice guidelines and clinical practice recommendations for 2006 updates: hemodialysis adequacy, peritoneal dialysis adequacy and vascular access. Am J Kidney Dis 2006;48(Suppl 1):S1-S322

3 Whittier WL. Surveillance of hemodialysis vascular access. Semin Intervent Radiol 2009;26(2):130-138

4 Teodorescu V, Gustavson S, Schanzer H. Duplex ultrasound evaluation of hemodialysis access: a detailed protocol. Int J Nephrol 2012;2012:508956

5 Krivitski NM. Novel method to measure access flow during hemodialysis by ultrasound velocity dilution technique. ASAIO J 1995; 41(3):M741-M745

6 Krivitski NM. Theory and validation of access flow measurement by dilution technique during hemodialysis. Kidney Int 1995;48(1): 244-250

7 Lindsay RM, Blake PG, Malek P, Posen G, Martin B, Bradfield E. Hemodialysis access blood flow rates can be measured by a differential conductivity technique and are predictive of access clotting. Am J Kidney Dis 1997;30(4):475-482

8 Schneditz D, Fan Z, Kaufman A, Levin NW. Measurement of access flow during hemodialysis using the constant infusion approach. ASAIO J 1998;44(1):74-81

9 Lindsay RM, Bradfield E, Rothera C, Kianfar C, Malek P, Blake PG. A comparison of methods for the measurement of hemodialysis access recirculation and access blood flow rate. ASAIO J 1998; 44(1):62-67

10 Yarar D, Cheung AK, Sakiewicz P, et al. Ultrafiltration method for measuring vascular access flow rates during hemodialysis. Kidney Int 1999;56(3):1129-1135

11 Asif A, Leon C, Orozco-Vargas LC, et al. Accuracy of physical examination in the detection of arteriovenous fistula stenosis. Clin J Am Soc Nephrol 2007;2(6):1191-1194

12 Leon C, Orozco-Vargas LC, Krishnamurthy G, et al. Accuracy of physical examination in the detection of arteriovenous graft stenosis. Semin Dial 2008;21(1):85-88

13 Wu S, Kalva SP. Dialysis Access Management. Switzerland: Springer; 2015

14 Rajan DK, ed. Essentials of Percutaneous Dialysis Interventions. New York: Springer; 2011

15 Sands J. The role of color-flow Doppler ultrasound in the management of hemodialysis accesses. ASAIO J 1998;44(1):41-43

16 Strauch BS, O'Connell RS, Geoly KL, Grundlehner M, Yakub YN, Tietjen DP. Forecasting thrombosis of vascular access with Doppler color flow imaging. Am J Kidney Dis 1992;19(6):554-557

17 Finlay DE, Longley DG, Foshager MC, Letourneau JG. Duplex and color Doppler sonography of hemodialysis arteriovenous fistulas and grafts. Radiographics 1993;13(5):983-989

18 Besarab A, Sherman R. The relationship of recirculation to access blood flow. Am J Kidney Dis 1997;29(2):223-229

19 Besarab A, Sullivan KL, Ross RP, Moritz MJ. Utility of intra-access pressure monitoring in detecting and correcting venous outlet stenoses prior to thrombosis. Kidney Int 1995;47(5): 1364-1373

20 Sullivan KL, Besarab A, Bonn J, Shapiro MJ, Gardiner GA Jr, Moritz MJ. Hemodynamics of failing dialysis grafts. Radiology 1993; 186(3):867-872

21 Magnasco A, Alloatti S, Martinoli C, Solari P. Glucose pump test: a new method for blood flow measurements. Nephrol Dial Transplant 2002;17(12):2244-2248

22 Steuer RR, Miller DR, Zhang S, Bell DA, Leypoldt JK. Noninvasive transcutaneous determination of access blood flow rate. Kidney Int 2001;60(1):284-291

23 Gotch FA, Buyaki R, Panlilio F, Folden T. Measurement of blood access flow rate during hemodialysis from conductivity dialysance. ASAIO J 1999;45(3):139-146

24 Mercadal L, Hamani A, Béné B, Petitclerc T. Determination of access blood flow from ionic dialysance: theory and validation. Kidney Int 1999;56(4):1560-1565

25 Weitzel WF, Rubin JM, Swartz RD, Woltmann DJ, Messana JM. Variable flow Doppler for hemodialysis access evaluation: theory and clinical feasibility. ASAIO J 2000;46(1):65-69

26 Weitzel WF, Rubin JM, Leavey SF, Swartz RD, Dhingra RK, Messana JM. Analysis of variable flow Doppler hemodialysis access flow measurements and comparison with ultrasound dilution. Am J Kidney Dis 2001;38(5):935-940

27 Goldstein SL, Allsteadt A. Ultrasound dilution evaluation of pediatric hemodialysis vascular access. Kidney Int 2001;59(6):2357-2360

28 Depner TA, Krivitski NM. Clinical measurement of blood flow in hemodialysis access fistulae and grafts by ultrasound dilution. ASAIO J 1995;41(3):M745-M749

29 Krivitski NM, MacGibbon D, Gleed RD, Dobson A. Accuracy of dilution techniques for access flow measurement during hemodialysis. Am J Kidney Dis 1998;31(3):502-508

30 Gray RJ, Sands JJ. Dialysis Access: A Multidisciplinary Approach. Philadelphia: Lippincott Williams \& Wilkins; 2002

31 Berman SS. Vascular Access in Clinical Practice. Boca Raton, FL: CRC Press; 2002

32 Henrich WL. Principles and Practice of Dialysis. Philadelphia: Lippincott Williams \& Wilkins; 2012

33 Ronco C, Levin NW. Hemodialysis, Vascular Access, and Peritoneal Dialysis Access. Vol 142. Switzerland: Karger Medical and Scientific Publishers; 2004 